\title{
FUNDAMENTAL SOLUTIONS AND HARMONIC ANALYSIS ON NILPOTENT GROUPS
}

\author{
A. NAGEL, F. RICCI, AND E.M. STEIN
}

Our purpose here is to announce results in harmonic analysis related to a large class of hypoelliptic operators on arbitrary simply-connected nilpotent Lie groups. We find the asymptotic development of their fundamental solutions, both locally and at infinity, and study corresponding Riesz transforms and analogues of the Hardy-Littlewood-Sobolev theorem for fractional integration. Details will appear elsewhere (see [NRS]).

For linear partial differential operators $P=\sum_{|\alpha| \leq m} a_{\alpha}(x) D^{\alpha}$ on domains in $\mathbf{R}^{n}$, a classical approach to studying existence and regularity of solutions is the procedure of freezing coefficients: for each point $x_{0}$ one approximates the operator $P$ by the constant coefficient operator $P_{x_{0}}=\sum_{|\alpha|=m} a_{\alpha}\left(x_{0}\right) D^{\alpha}$. The operator $P_{x_{0}}$ is then a translation invariant operator on the group $\mathbf{R}^{n}$, and at least when $P$ is elliptic, $P_{x_{0}}$ can be inverted and its inverse studied by using the harmonic analysis of the Abelian Lie group $\mathbf{R}^{n}$. This is essentially the idea of the usual calculus of pseudo-differential operators. For nonelliptic operators, one cannot use this simple procedure, but starting with the work of $[\mathrm{FoS}]$ and $[\mathrm{RoS}]$ it is now known how to analyze certain hypoelliptic operators $P$ by approximating them at each point by a left invariant differential operator on an appropriate nilpotent Lie group.

These operators all arise as polynomials in real vector fields, where the vector fields are assumed to satisfy the Hörmander [H] condition that the Lie algebra they generate spans the tangent space at each point. The method used is to model the algebra generated by these vector fields by the Lie algebras of certain nilpotent Lie groups. Further analysis allowed one to determine at least the approximate size of the fundamental solutions of these operators (see [NSW]). Thus the idea of approximating by nilpotent groups

Received by the editors May 30, 1989 and, in revised form, December 8, 1989.

1980 Mathematics Subject Classification (1985 Revision). Primary 35A08, 22E25, 42B70. 
leads naturally to the question of finding precise descriptions of these fundamental solutions-and their resulting properties-in the case of left-invariant operators on general nilpotent Lie groups. This is the goal of our present work.

There are two ways of arriving at versions of the basic operators in question. If we take the harmonic analysis of the usual Laplacian $\Delta$ on $\mathbf{R}^{n}$ as our model, then one approach is an extrinsic one. The fundamental solution of $\Delta$ is the potential $K_{n}(x)=c_{n}|x|^{-n+2}$ (at least when $n>2$ ), and for suitable $f$ the solution to the equation $\Delta(u)=f$ is given by $f * K_{n}$. The extrinsic approach proceeds by studying general convolution operators $f \rightarrow f * K$, where $K$ is a Calderón-Zygmund kernel, or a kernel displaying a more general homogeneity on $\mathbf{R}^{n}$.

A second approach is an intrinsic one. Here one proceeds to directly study functions of the operator $\Delta$ which can be defined by using the spectral theorem or the Fourier transform. These functions include the Riesz transforms,

$$
\frac{\partial}{\partial x_{j}}(-\Delta)^{-1 / 2}, \quad \frac{\partial^{2}}{\partial x_{i} \partial x_{j}}(-\Delta)^{-1},
$$

and the fractional integration operators $\Delta^{-\alpha / 2}$.

For $\mathbf{R}^{n}$ these two approaches lead to essentially the same classes of operators. Both classes of operators commute appropriately with the dilations $x \rightarrow \delta x, \delta>0$, since these dilations are automorphisms of the group $\mathbf{R}^{n}$. The situation is, broadly speaking, the same when $\mathbf{R}^{n}$ is replaced by a nilpotent group which carries automorphic dilations and when $\Delta$ is replaced by a corresponding homogeneous operator. However, the situation changes markedly in the situation of a general nilpotent Lie group; then the operators that arise in these two classes are essentially different. The study of singular integrals (the extrinsic approach) was carried out in [RiS 1] and [RiS 2].

Here we turn to the intrinsic approach on a general simply connected nilpotent Lie group $G$. The framework in which this is done is to assign to each suitable partial differential operator $\mathscr{L}$ on $G$, two other nilpotent Lie groups, $G_{0}$ and $G_{\infty}$ which arise from $G$ by contraction. These two groups have the feature that they do have automorphic dilations. What is crucial is that one can show that $G_{0}$ controls the local behavior of the fundamental solution of $\mathscr{L}$, and similarly $G_{\infty}$ plays an analogous role at infinity. 
We deal with the following operators $\mathscr{L}$. Let $X_{1}, X_{2}, \ldots, X_{k}$ be a set of left-invariant vector fields on $G$ which generate the Lie algebra $\mathfrak{g}$ of $G$. Let $\mathscr{L}=\mathscr{P}\left(X_{1}, X_{2}, \ldots, X_{k}\right)$ be a noncommutative polynomial in $X_{1}, X_{2}, \ldots, X_{k}$, which is homogeneous of degree $d$, once we assign degrees $a_{1}, a_{2}, \ldots, a_{k}$ to $X_{1}, X_{2}, \ldots, X_{k}$, respectively; we also assume that $\mathscr{P}$ is universally hypoelliptic (see below). Examples of such $\mathscr{L}$ are $\sum_{j=1}^{k-1}(-1)^{b_{j}} X_{j}^{2 b_{j}}+X_{k}$, where $b_{j}$ are positive integers (here $2 a_{j} b_{j}=$ $\left.d=a_{k}, j=1, \ldots, k-1\right)$; also systems $\left\{\mathscr{L}_{j}\right\}$, such as $\mathscr{L}_{j}=$ $X_{j}, j=1, \ldots, k$.

\section{Main Results}

A feature of our construction is that the homogeneous groups $G_{0}$ and $G_{\infty}$ are realized on the same space as $G$. Let $Q_{0}$ (and $Q_{\infty}$ ) denote their respective homogeneous dimensions. To $\mathscr{L}$ we associate variants $\mathscr{L}_{0}$ (and $\mathscr{L}_{\infty}$ ) on $G_{0}$ (and $G_{\infty}$ ), each homogeneous of degree $d$. This is done as follows. The construction of $\mathfrak{g}_{0}$ (see below) actually corresponds to a choice of a gradation in $\mathfrak{g}$, i.e. $\mathfrak{g}=\sum_{j} \oplus \mathfrak{v}_{j}$, where the elements in $\sum_{j \leq k} \oplus \mathfrak{v}_{j}$ have degree less than or equal to $k$ in the above sense. Let $p_{j}$ denote the projection on $\mathfrak{v}_{j}$, and set $\bar{X}_{j}=p_{j}\left(X_{j}\right), \mathscr{L}_{0}=\mathscr{P}\left(\bar{X}_{1}, \ldots, \bar{X}_{k}\right)$. There is a similar definition for $\mathscr{L}_{\infty}$. Note that this construction may depend on the particular realization of $\mathscr{L}$ as a polynomial in $X_{1}, \ldots, X_{k}$. We use the notion of a regular quasi-homogenous function of degree $r$ on $G_{0}$ (or $G_{\infty}$ ) to be a function which is $C^{\infty}$ away from the origin and is homogeneous of degree $r$; and if $r$ is an integer we allow also terms of the form $Q \log \rho$, where $Q$ is a polynomial of degree $r$ and $\rho$ is a smooth homogeneous norm function.

Theorem 1. $\mathscr{L}$ has a fundamental solution, which when suitably normalized has the following complete asymptotic expansion:

(a) Near the origin,

$$
K(x) \sim \sum_{m=0}^{\infty} K_{m}(x), \quad x \rightarrow 0,
$$

where $K_{m}$ is a regular quasi-homogeneous function of degree $-Q_{0}+d+m$, and $K_{0}$ is the fundamental solution of the operator $\mathscr{L}_{0}$ on $G_{0}$. 
(b) Near infinity,

$$
K(x) \sim \sum_{m=0}^{\infty} \bar{K}_{m}(x), \quad x \rightarrow+\infty,
$$

where $\bar{K}_{m}$ has degree $-Q_{\infty}+d+m$, and $\bar{K}_{0}$ is the fundamental solution of $\mathscr{L}_{\infty}$ on $G_{\infty}$.

Remark. On homogeneous groups such as $G_{0}, G_{\infty}$, the existence of $K_{0},\left(\bar{K}_{0}\right)$ follows from the work of Folland [F] and Geller [Ge].

The applications are as follows. $\left\{\mathscr{L}_{j}\right\}$ is a system as above, of operators homogeneous of degree $d . \mathscr{R}=Q\left(X_{1}, \ldots, X_{k}\right)$ is a homogeneous monomial of degree $d^{\prime}, d^{\prime} \leq d$. Part (a) of the following theorem is motivated by [V].

\section{Theorem 2.}

(a) If $0 \leq d^{\prime}<d$, we have the a priori inequality

$$
\|\mathscr{R}(f)\|_{L^{q}(G)} \leq C \sum_{j}\left\|\mathscr{L}_{j}(f)\right\|_{L^{p}(G)}
$$

for all $f \in C_{0}^{\infty}(G)$, if $1<p, q<\infty$, as long as

$$
\frac{d-d^{\prime}}{Q_{\infty}} \leq \frac{1}{p}-\frac{1}{q} \leq \frac{d-d^{\prime}}{Q_{0}} .
$$

(b) If $d=d^{\prime}$, the inequalities hold for $q=p, 1<p<\infty$.

Sketch of proof. We discuss briefly some features of the proof. The groups $G_{0}$ and $G_{\infty}$ arise as follows. Suppose $\mathscr{F}$ is the free nilpotent Lie algebra with generators $Y_{1}, \ldots, Y_{k}$, of sufficiently high step. Then the mapping $Y_{j} \rightarrow X_{j}, j=1, \ldots, k$, extends to an isomorphism of $\mathscr{F} / \mathfrak{i}$ with $\mathfrak{g}$, where $\mathfrak{i}$ is a suitable ideal in $\mathscr{F}$. On $\mathscr{F}$ we can define (automorphic) dilations $D_{\delta}, 0<\delta<\infty$, by

$$
D_{\delta}\left(Y_{j}\right)=\delta^{a_{j}} Y_{j}, \quad j=1, \ldots, k .
$$

We then set $\mathfrak{i}_{\delta}=D_{\delta}^{-1}(\mathfrak{i})$, and use the fact that $\mathfrak{i}_{\delta}$ tend to limiting ideals $\mathfrak{i}_{0}$ and $\mathfrak{i}_{\infty}$ as $\delta \rightarrow 0$ or $\delta \rightarrow \infty$. (See also [Go] and [HuJ]). Then $\mathfrak{g}_{0}=\mathscr{F} / \mathfrak{i}_{0}$, and $\mathfrak{g}_{\infty}=\mathscr{F} / \mathfrak{i}_{\infty}$ are the Lie algebras of $G_{0}$ and $G_{\infty}$, respectively. We assume that $\mathscr{P}$ is universally hypoelliptic in the sense that the operator $\mathscr{P}\left(Y_{1}, \ldots, Y_{k}\right)$ is a hypoelliptic 
differential operator on $\mathscr{F}$. Next, at least heuristically,

$$
\begin{gathered}
K(x)=\int_{i} \widetilde{K}(x+t) d t ; \quad K_{0}(x)=\int_{i_{0}} \widetilde{K}(x+t) d t \\
\bar{K}_{0}=\int_{i_{\infty}} \widetilde{K}(x+t) d t .
\end{gathered}
$$

where $\widetilde{K}$ is the fundamental solution corresponding to a suitably lifted operator $\widetilde{\mathscr{L}}$ on $\mathscr{F}$. (This is motivated by [RoS] and [NSW].)

The main difficulties in dealing with (1) are twofold. First, the integrals above do not converge in general and must be suitably regularized. Secondly, one needs to see why the leading terms of the asymptotic expansion of the first integral give the second (or third) integral at the origin (or at infinity).

\section{REFERENCES}

[F] G.B. Folland, Sub-elliptic estimates and function spaces on nilpotent groups, Ark. Mat. 13 (1975), 161-207.

[FoS] G.B. Folland and E.M. Stein, Estimates for the $\bar{\partial}_{b}$ complex and analysis of the Heisenberg group, Comm. Pure Appl. Math. 27 (1974), 429-522.

[Ge] D. Geller, Liouville's theorem for homogeneous groups, Comm. Partial Differential Equations 15 (1983), 1665-1677.

[Go] R. Goodman, Nilpotent lie groups, Lecture Notes in Math., vol. 562, Springer-Verlag, New York, 1976.

[H] L. Hörmander, Hypoelliptic second order differential equations, Acta Math. 119 (1967), 147-171.

[HuJ] A. Hulanicki and J. Jenkins, Nilpotent Lie groups and summability of eigenfunction expansions of Schrödinger operators, Studia Math. 80 (1984), 235244.

[NRS] A. Nagel, F. Ricci and E.M. Stein, Harmonic analysis and fundamental solutions on nilpotent Lie groups, in Festshrift in honor of Mischa Cotlar (to appear).

[NSW] A. Nagel, E.M. Stein and S. Wainger, Balls and metrics defined by vector fields, Acta Math. 155 (1985), 103-147.

[RiS1] R. Ricci and E.M. Stein, Oscillatory singular integrals and harmonic analysis on nilpotent groups, Proc. Nat. Acad. Sci. U.S.A. 83 (1986), 1-3.

[RiS2] F. Ricci and E.M. Stein, Harmonic analysis on nilpotent groups and singular integrals II, J. Funct. Anal. 78 (1988), 56-84. 
[RoS] L. Rothschild and E.M. Stein, Hypoelliptic differential operators and nilpotent groups, Acta Math. 137 (1976), 247-320.

[V] N. Varopolous, J. Funct. Anal. 66 (1986), 406-431.

Department of Mathematics, Princeton University, Princeton, New JERSEY 08540

Department of Mathematics, Politechnico of Torino, Corso Duca DEGLI ABRUZZI 24, 10129 TORIN, ITALY

Department of Mathematics, University of Wisconsin, Madison, WisCONSIN 53706 